\title{
Systematic review and meta-analysis of the safety and effectiveness of low molecular heparin for severe acute pancreatitis
}

\author{
Hui Wang, Ying Xu, Meijuan Yang, Dexing Zheng, Lijiao Chen \\ Department of Vascular Surgical Hernia and Abdominal Surgery, Taizhou Hospital of Zhejiang Province affiliated with Wenzhou Medical University, \\ Taizhou, China \\ Contributions: (I) Conception and design: H Wang, L Chen; (II) Administrative support: Y Xu; (III) Provision of study materials or patients: H Wang, \\ Y Xu, M Yang, D Zheng; (IV) Collection and assembly of data: All authors; (V) Data analysis and interpretation: H Wang, M Yang, D Zheng, L \\ Chen; (VI) Manuscript writing: All authors; (VII) Final approval of manuscript: All authors. \\ Correspondence to: Lijiao Chen. No. 150 Ximen Street, Linhai West City, Taizhou, China. Email: chenlijiao@enzemed.com.
}

\begin{abstract}
Background: Because the effect of low molecular heparin (LMH) on acute ulcer and bleeding complications in patients with severe acute pancreatitis (SAP) is unclear, we investigated the safety and efficacy of early intervention with $\mathrm{LMH}$ in patients with SAP.
\end{abstract}

Methods: Using the keywords "heparin", "low molecular weight heparin", "pancreatitis", and "severe acute pancreatitis", we searched PubMed, Medline, CNKI, etc. And select the reference documents of the comparative study of traditional treatment and low molecular weight heparin intervention. RevMan was used for the meta-analysis.

Results: A total of 8 references were included in the study, and most of them were low risk bias (medium and high quality). Meta-analysis shows that, The MHS between the two groups is statistically heterogeneous. $\left(\mathrm{Chi}^{2}=19.59, \mathrm{I}^{2}=95 \%, \mathrm{P}<0.00001\right)$, Fixed-effects model $(\mathrm{FEM})$ analysis showed that the MHS of experimental subjects was obviously shorter than that of controls $(\mathrm{Z}=3.24, \mathrm{P}=0.001)$; The acute physiology and chronic health score (APACHE II) of the two groups were heterogeneous $\left(\mathrm{Chi}^{2}=7.24, \mathrm{I}^{2}=72 \%, \mathrm{P}=0.03\right.$ ); No heterogeneity was found in the amount of bleeding $\left(\mathrm{Chi}^{2}=5.83, \mathrm{I}^{2}=31 \%, \mathrm{P}=0.21\right), \mathrm{FEM}$ analysis showed the number of complications in the experimental group was significantly less than that in the control group $(\mathrm{Z}=2.70, \mathrm{P}=0.007)$.

Discussion: LMH intervention can dramatically reduce the average hospital stay and complications of patients with SAP, improve treatment efficacy, and has high safety.

Keywords: APACHE II score; low molecular heparin (LMH); severe acute pancreatitis

Submitted Sep 09, 2021. Accepted for publication Nov 03, 2021.

doi: 10.21037/apm-21-3058

View this article at: https://dx.doi.org/10.21037/apm-21-3058

\section{Introduction}

Severe acute pancreatitis (SAP) is a severe type of acute pancreatitis, accompanied by organ dysfunction, or local complications such as necrosis, abscess or pseudocyst, or both (1). After acute pancreatitis, pancreatic juice leaks out and is wrapped by surrounding fibrous tissue, forming a cyst without endothelial cells, called pseudocyst. It begins to form in the early stage of pancreatitis, and the pseudocyst wall is relatively complete one month after the onset of pancreatitis, and this type of cyst is mostly behind the stomach wall $(2,3)$. Conservative treatment should be performed firstly. Somatostatin, which inhibits the secretion of pancreatic juice, can be used. Some cysts can gradually disappear. If it does not resolve, internal drainage can be performed, or cyst puncture and drainage under gastroscopy. In the 1980s, most cases of severe acute 
pancreatitis died in the early stages of the disease. Until recent years, with the progress of SAP surgical treatment, the cure rate has increased, but the overall mortality rate is still high. The cause of pancreatitis varies from region to region $(4,5) .70 \%$ to $80 \%$ of severe acute pancreatitis is caused by biliary diseases, alcoholism, and overeating (6). The treatment of severe acute pancreatitis is mainly based on correcting water and electrolyte disorders, inhibiting inflammatory reactions and preventing complications. Studies have found that somatostatin and its analogues can directly inhibit pancreatic exocrine secretion and play a positive role in preventing pancreatitis after endoscopic retrograde cholangiopancreatography (7).

Heparin, or heparin sodium or heparin calcium, is an anticoagulant first found in the liver. It is commonly used in injectable to prevent diffuse intravascular coagulation caused by diseases of thrombosis or embolism and other causes $(8,9)$. Low molecular heparin ( $\mathrm{LMH})$ has an anticoagulant effect in vivo and in vitro, and the effect is mediated by antithrombin III. Antithrombin activity depends on the presence of specific pentose sequences with a high affinity for antithrombin III and the number of monosaccharide chains $(10,11)$.

The etiology of SAP is complex. Many studies have revealed that pancreatic microcirculation disorders play an active part in the pathogenesis of acute pancreatitis, promoting bleeding and necrosis of the pancreas and playing a role in sustained damage during the whole development process of acute pancreatitis $(12,13)$. Even with current treatments, the fatality rate of SAP remains high, with multiple organ damage. Promoting the patency of capillaries and reducing thrombosis are of great significance for the prognosis and mortality of pancreatitis $(14,15)$. Acute pancreatitis can be treated with low doses of LMH and insulin, or rapid lipid-lowering with lipid adsorption and plasmapheresis (16). Continuous intravenous infusion of small doses of heparin and insulin (17) can stimulate the activity of lipoprotein enzymes, accelerate the degradation of chylomicrons, and effectively reduce triglyceride levels (18). Vomiting and large amounts of inflammatory exudation in patients with SAP lead to decreased circulating blood volume and increased blood viscosity, resulting in microthrombi formation in the pancreas and exacerbation of symptoms. Li et al. (2020) pointed out that in acute pancreatitis with hyperlipidemia, triglycerides needed to be reduced to $<5.65 \mathrm{mmoL} / \mathrm{L}$ in a short period of time (19). Measures included LMH $5000 \mathrm{U}$ subcutaneous injection every $12 \mathrm{~h}$ to increase lipoprotein enzyme activity and accelerate the degradation of chylomicrons (20).

Low molecular heparin ( $\mathrm{LMH}$ ) can improve microcirculation perfusion, fight against the body's hypercoagulable state, improve pancreatic microcirculation, reduce the level of inflammatory factors in the body, and prevent further ischemic necrosis. However, according to current research, the impact of LMH on the occurrence of emergency ulcers and bleeding complications in patients with SAP after anticoagulation therapy is still unclear. Therefore, this study adopted the meta-analysis method to improve the efficiency of statistical analysis, conducted a combined analysis of multiple similar research results, and increased the sample size and improved the test efficiency from a statistical point of view, so as to explore the safety and effectiveness of LMH in SAP. We present our study in accordance with the PRISMA reporting checklist (available at https://dx.doi.org/10.21037/apm-21-3058).

\section{Methods}

\section{Literature search}

We used the terms "heparin", "low molecular weight heparin", "pancreatitis", and "severe acute pancreatitis" to search the online literature databases of PubMed, Medline, Embase, China Biomedical Literature, CNKI, Wanfang, VIP, and Google Scholar for relevant studies published up to October 30, 2020. Studies that were not indexed by the database were screened by tracing all of the included reference lists. RevMan 5.2 provided by the Cochrane system was used for quality assessment. Through reading topics and abstracts, the initially retrieved documents are screened for the first time, the non-conforming documents are excluded, and the included documents are determined. According to the inclusion and exclusion criteria, the second screening was conducted, and search engines were used to trace the included documents. Finally, through reading the full text of the literature, the third screening is carried out to evaluate the quality of the articles.

\section{Inclusion and exclusion criteria}

Our inclusion and exclusion criteria were as follows.

Inclusion criteria: (I) diagnosis of SAP, without restriction of pathological type; (II) intervention measures for controls comprised conventional treatment (including fluid resuscitation, trypsin inhibitors, antibiotics, and symptomatic treatment), but no heparin intervention; (III) 
experimental subjects received LMH intervention; (IV) randomized clinical trial, prospective cohort and casecontrol study.

Exclusion criteria: (I) patients with infectious or neurological diseases; (II) not a randomized control trial; (III) lack of valid data; (IV) overlapping research subjects or data; (V) duplicate publication or with insufficient experimental subjects.

\section{Quality assessment}

Pathological control studies were assessed by the NewcastleOttawa Scale (NOS) of the Cochrane Collaboration. A nine-star system assessed the results and comparisons: $\geq 7$ stars suggested a high-quality study (low risk bias); $\leq 1$ star indicated low-quality (high risk bias) study; 2-6 stars suggested medium quality.

Two senior experts independently assessed the quality of the studies, and conducted three preliminary tests. Any disagreement between experts was resolved by consensus after discussion or with arbitration by a third expert.

\section{Data extraction}

A unified Excel was utilized to extract data by the two experts independently, with three preliminary tests. Any disagreements between experts were resolved as described above. The extracted data comprised: (I) first author and publication year; (II) number of subjects; (III) groupings and the interventions adopted for both groups; (IV) recovery indicators before and after treatment, such as the acute physiology and chronic health score (APACHE II), prothrombin time (PT), pancreatic pseudocyst occurrence, mean hospital stay (MHS), and death.

\section{Statistical analysis}

The Review manager5.3 software was adopted for Metaanalysis. Mean deviation (MD) or standard mean deviation (SMD) and 95\% confidence interval (CI) were used for the continuous variables of the study data as the efficacy analysis statistics. The included articles were tested for heterogeneity ( $\mathrm{Q}$ test). The risk of bias assessment chart of Reviw Manager software was used to assess the risk bias of the included articles. Each effect was expressed using a $95 \%$ CI. When $\mathrm{P}>0.1$ and $\mathrm{I}^{2}<50 \%$, the fixed effects model was used for meta-analysis. When $\mathrm{P}<0.1$ and $\mathrm{I}^{2}>50 \%$, the random effects model was used for meta-analysis.

\section{Results}

\section{Literature screening results}

As shown in Figure 1, a total of 526 studies was obtained, of which 300 were eliminated by abstract and title, and 212 were eliminated after reading the whole article. Finally, 8 studies were available for the meta-analysis. The excluded literature mainly included the existence of primary acute and chronic cardiopulmonary dysfunction and serious mental diseases (34 articles); animal experiment (30 articles); no relevant information was retrieved (37 articles); data unavailable (51 articles); and lack of original data (66 articles). Table 1 shows the basic information of the included studies, which covered the years 2002-2016. Figure 2 shows the rating results of the NOS scale: there were $25 \%$ studies with $\geq 7$ stars, and $75 \%$ with $2-6$ stars, meaning all were mediumto high-quality studies.

\section{Results of risk bias evaluation of the literature}

Figures 3,4 show that each methodological feature was included. Random sequence generation and allocation hiding (selection bias), blinding of results (measurement bias), incomplete results data (follow-up bias), and selective reporting (reporting bias) were low. Moreover, subjects' and researchers' blinding (i.e., implementation bias), as well as other low-risk bias evaluations were $\sim 50 \%$. Apart from Barkay [2008] (1) and Rabenstein et al. [2002] (4), the bias of all the other studies was dramatically low risk.

\section{Prothrombin time}

Figure 5 shows the comparison of PT between groups after treatment. Lu et al.'s (7) results accounted for the highest part $(35.1 \%)$. The horizontal line (HL) of the $95 \%$ CI of most studies was on the left of the invalid vertical line (IVL), which crossed the HL. The HL of $95 \%$ CI of a few studies was on the right of the IVL. There were 293 controls and 299 experimental subjects. The comparison of PT between groups was heterogeneous $\left(\mathrm{Chi}^{2}=17.19, \mathrm{I}^{2}=88 \%, \mathrm{P}=0.0002\right)$. The combined effect size (diamond block) was on the left side of the IVL [odds ratio (OR) $=0.05,95 \%$ CI: $-0.99,1.09$ ]. REM analysis showed no great difference in PT between groups $(\mathrm{Z}=0.09, \mathrm{P}=0.93)$.

Figure 6 is a funnel plot of PT between groups. The asymmetry of the circles on either side of the invalid midline indicated publication bias. 
Identification of studies via databases and registers
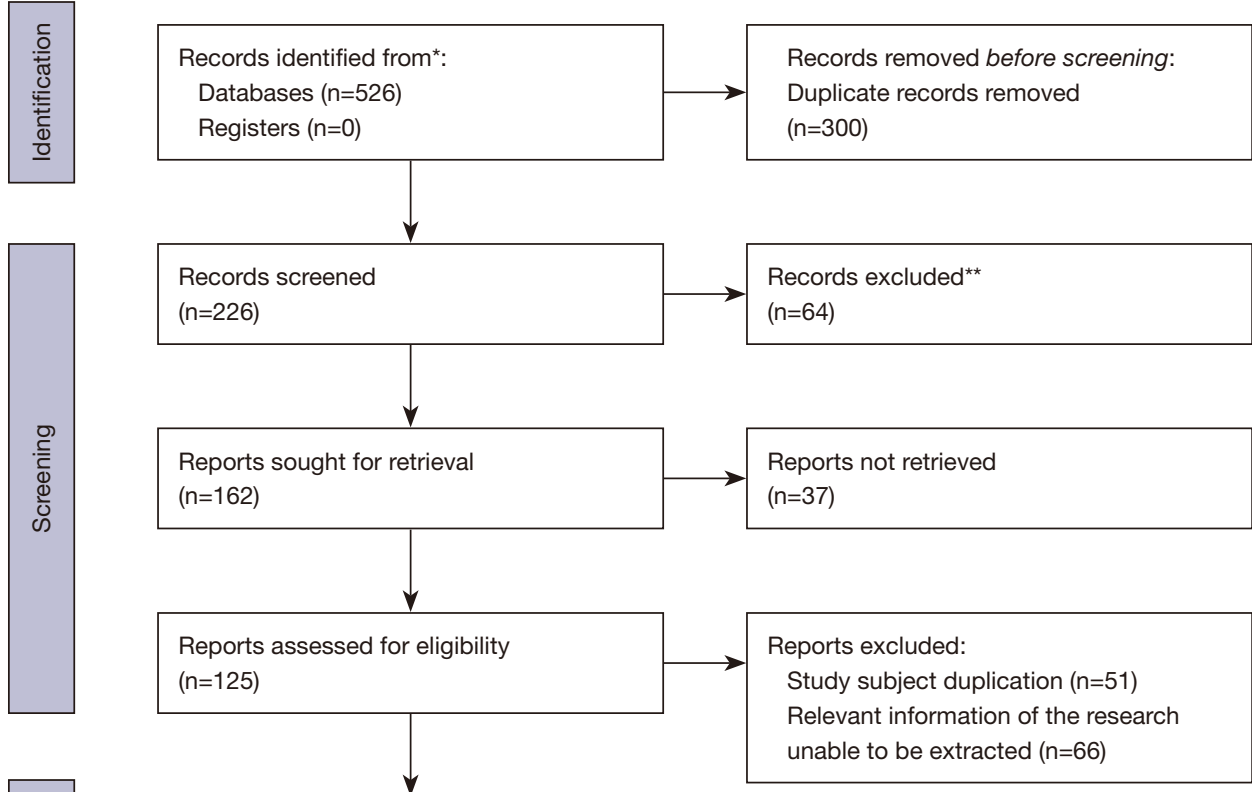

Studies included in review $(n=8)$

Reports of included studies $(n=0)$

Figure 1 Literature screening. *, representative databases and registers; ${ }^{* *}$, representative documents with incomplete information.

Table 1 Basic characteristics of the studies included in the meta-analysis

\begin{tabular}{|c|c|c|c|c|c|c|c|}
\hline Authors & $\begin{array}{c}\text { Year published } \\
\text { year }\end{array}$ & $\begin{array}{c}\text { Control } \\
\text { cases }\end{array}$ & $\begin{array}{c}\text { Experimental } \\
\text { cases }\end{array}$ & Control group & $\begin{array}{l}\text { Experimental } \\
\text { group }\end{array}$ & Parameters & $\begin{array}{l}\text { Intervention time } \\
\text { (weeks) }\end{array}$ \\
\hline Barkay et al. (1) & 2008 & 54 & 51 & $\begin{array}{c}\text { Conventional } \\
\text { treatment }\end{array}$ & LMH & Complications & \\
\hline Du et al. (2) & 2014 & 33 & 34 & $\begin{array}{l}\text { Conventional } \\
\text { treatment }\end{array}$ & $\mathrm{LMH}$ & PT and MHS & 2 \\
\hline Rabenstein et al. (4) & 2002 & 547 & 249 & $\begin{array}{l}\text { Conventional } \\
\text { treatment }\end{array}$ & LMH & Complications & \\
\hline Rabenstein et al. (5) & 2004 & 227 & 221 & $\begin{array}{c}\text { Conventional } \\
\text { treatment }\end{array}$ & LMH & Complications & \\
\hline He et al. (8) & 2016 & 32 & 34 & $\begin{array}{l}\text { High-volume } \\
\text { hemofiltration }\end{array}$ & LMH & APACHE II & \\
\hline
\end{tabular}

LMH, low molecular heparin; PT, prothrombin time; MHS, mean hospital stay. 


\section{Mean hospital stay}

Figure 7 shows the comparison results of the MHS between control group and experimental group. Among the 2 studies included, there were 163 controls, and 169 experimental subjects. The MHS between the two groups is statistically heterogeneous. $\left(\mathrm{Chi}^{2}=19.59, \mathrm{I}^{2}=95 \%, \mathrm{P}<0.00001\right)$. On the left side of the IVL was the diamond block (OR $=-9.88$, $95 \%$ CI: $-15.85,-3.90)$. FEM analysis showed that the MHS of experimental subjects was obviously shorter than that of controls $(\mathrm{Z}=3.24, \mathrm{P}=0.001)$.

Figure 8 is a funnel plot of the MHS for the two groups, showing high accuracy of the studies and no publication bias, because the circles were symmetrical on both sides of the midline toward the top of the funnel plot.

\section{Complications of two groups.}

Figure 9 shows the results of a comparison of complications

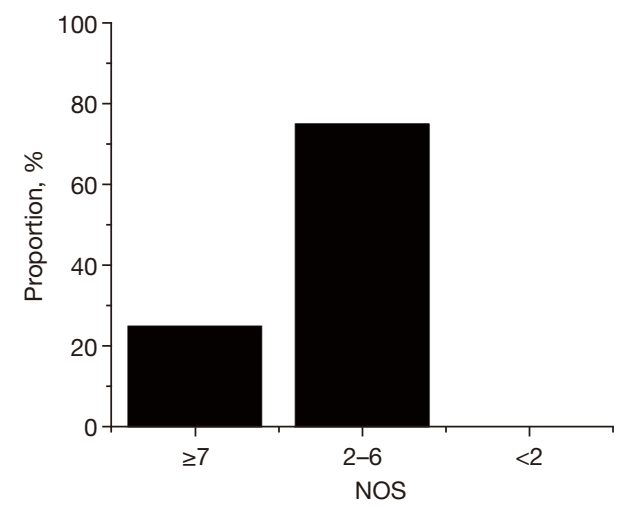

Figure 2 Quality rating of Newcastle-Ottawa Scale (NOS): $\geq 7$ and 2-6 indicate high-quality and medium-quality studies respectively. between groups. The results of Rabenstein et al. [2002] (4) accounted for the highest percentage (40.2\%), and second was Rabenstein et al. (28.8\%) (5). Most HL of the $95 \%$ CI were on the left of the IVL, which crossed the HL. There were 1,002 controls and 701 experimental subjects. No heterogeneity was found in the amount of bleeding $\left(\mathrm{Chi}^{2}=5.83, \mathrm{I}^{2}=31 \%, \mathrm{P}=0.21\right)$. On the left side of the IVL was the diamond block $(\mathrm{OR}=0.56,95 \% \mathrm{CI}: 0.37,0.85)$. FEM analysis showed the number of complications in the experimental group was significantly less than that in the control group $(\mathrm{Z}=2.70, \mathrm{P}=0.007)$.

Figure 10 is a funnel plot of complications between groups. The circles were symmetrical to the midline in the top of the plot, suggesting high accuracy and no publication bias.

\section{APACHE II scores of the two groups}

Figure 11 shows the APACHE II scores. The results of Lu et al. (7) [2010] accounted for a high percentage of the combined result (44.0\%). In addition, most HL of the $95 \%$ CI fell to the left of the IVL. There were 292 controls and 304 experimental subjects. The APACHE II scores of the two groups were heterogeneous $\left(\mathrm{Chi}^{2}=7.24, \mathrm{I}^{2}=72 \%\right.$, $\mathrm{P}=0.03)$. The diamond block was on the left of the IVL (OR $=-0.81,95 \%$ CI: $-1.70,0.08)$. REM analysis showed that there was no significant difference in APACHE II scores between the two groups $(\mathrm{Z}=1.79, \mathrm{P}=0.07)$.

Figure 12 is a funnel plot of the APACHE II scores in the two groups. The circles were not symmetrical on both sides of the midline, showing publication bias.

\section{Discussion}

The treatment of SAP can use low-dose LMH and

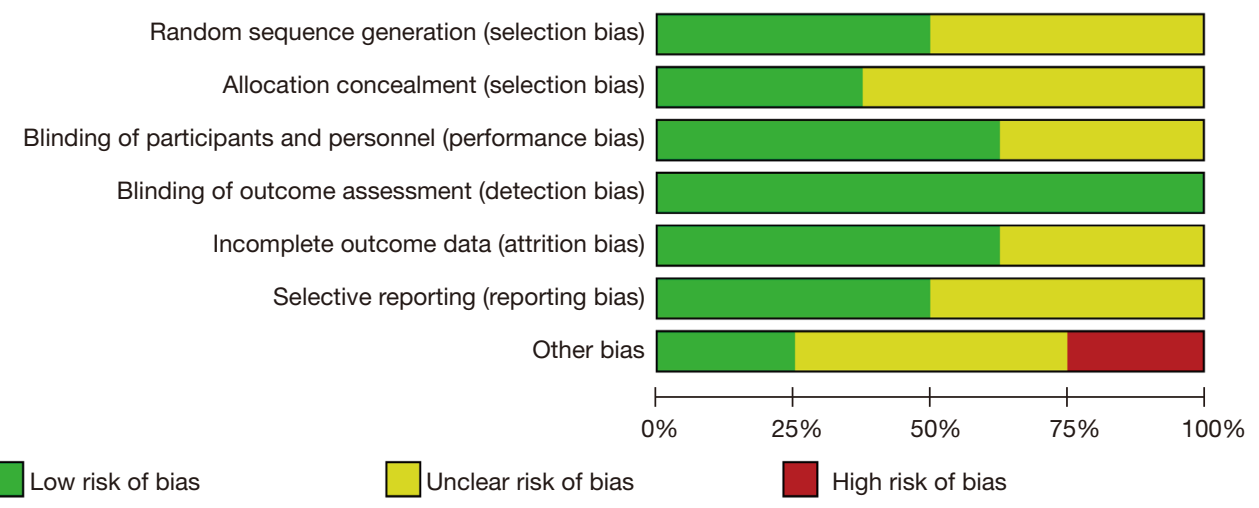

Figure 3 Risk bias evaluation. 


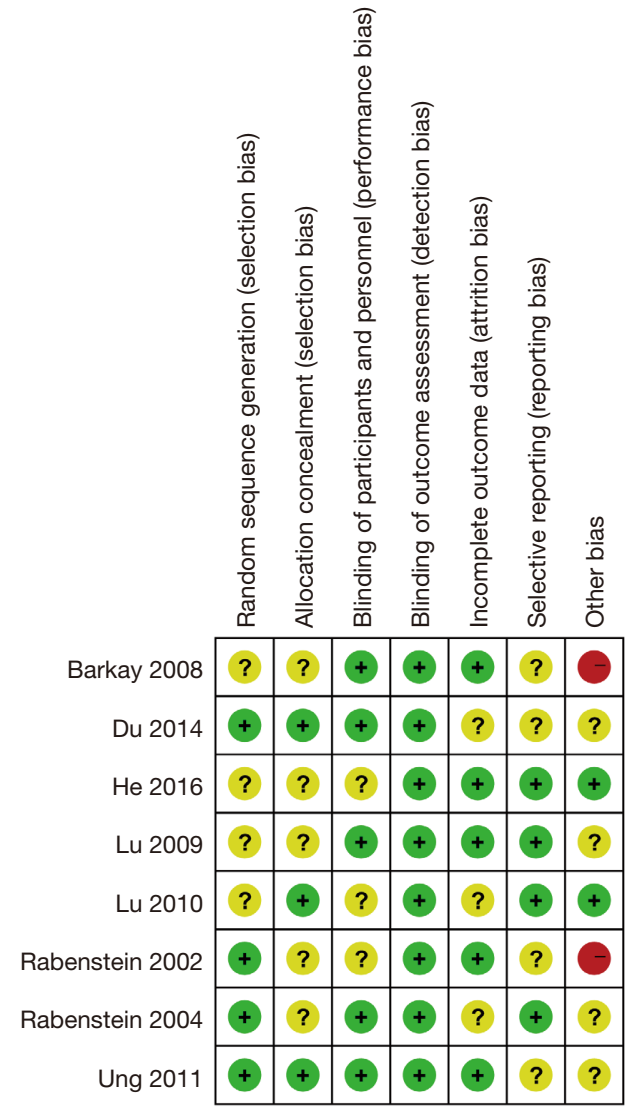

Figure 4 Bias-risk assessment diagram of the included articles. insulin, or lipid adsorption and plasma exchange to quickly lower lipids. Continuous intravenous infusion of small doses of heparin and insulin can stimulate the activity of lipoproteinases, accelerate the degradation of chylomicrons, and effectively reduce triglyceride levels. However, for patients with complications of abdominal hemorrhage after SAP surgery, anticoagulation therapy should be disabled. In addition, patients with bleeding tendency such as hypertensive intracranial hemorrhage, cerebral infarction, and active peptic ulcer should also be considered as contraindications for anticoagulation therapy. Meta-analysis was used in this study. Among the 14 papers included, 11 papers were grouped by random control, and only 3 papers were analyzed retrospectively, which brought bias to the study, but overall, it had little effect on the results of this study. A single sample study may be unstable. Meta-analysis is used to quantitatively synthesize all the included literatures in the study, which can not only avoid the differences among the studies that may be caused by the samples coming from different populations, but also give different weights to the results according to the sample size of each study, so as to increase the sample size and improve the credibility of the conclusions. The quality of meta-analysis mainly depends on the authenticity and completeness of the analyzed documents. Due to the objective influence of the included documents, the number

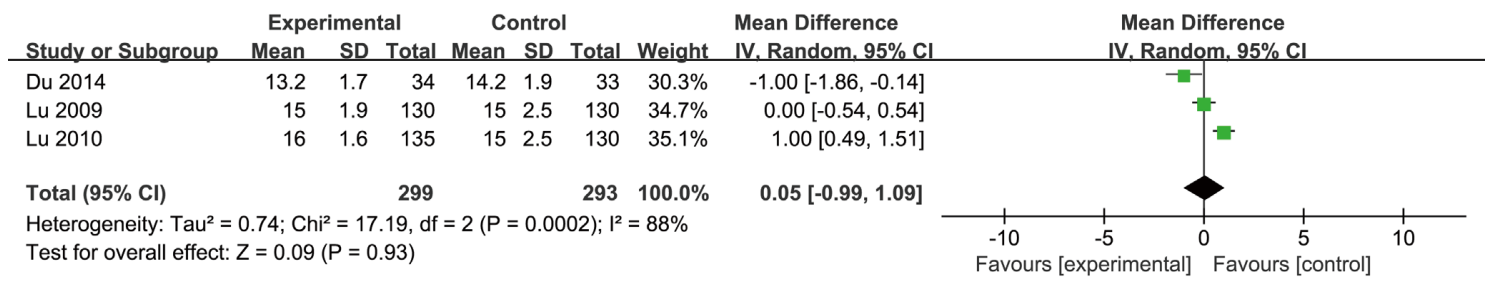

Figure 5 Prothrombin time.

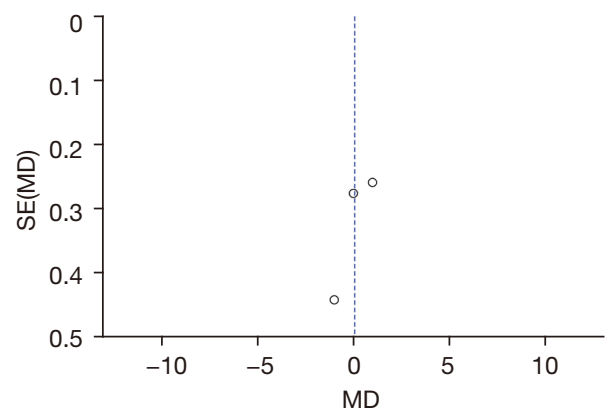

Figure 6 Funnel plot of prothrombin time. 


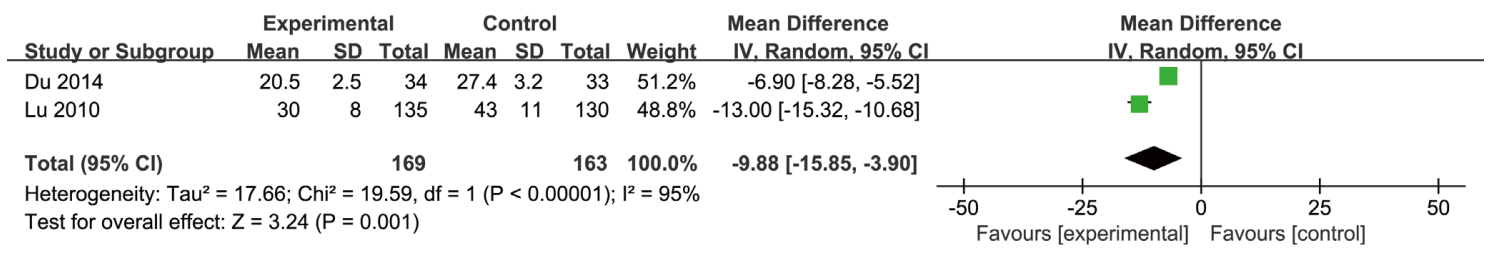

Figure 7 Mean hospital stay in the two groups.

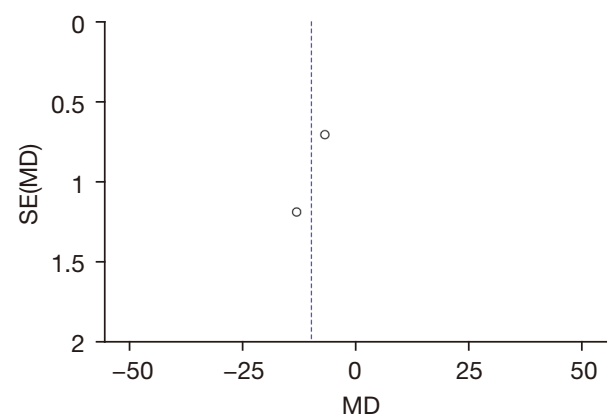

Figure 8 Funnel plot of mean hospital stay.

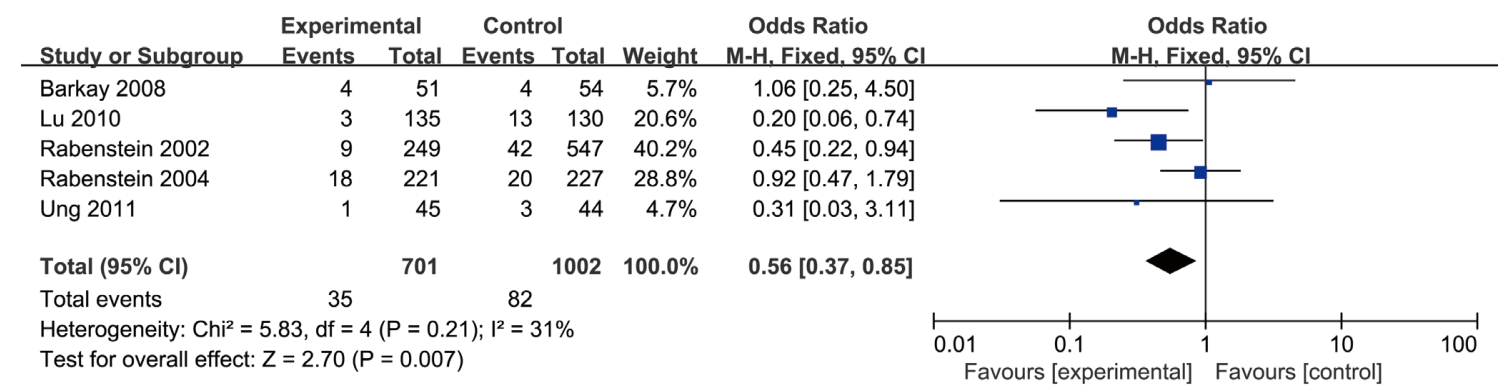

Figure 9 Comparison of blood loss.

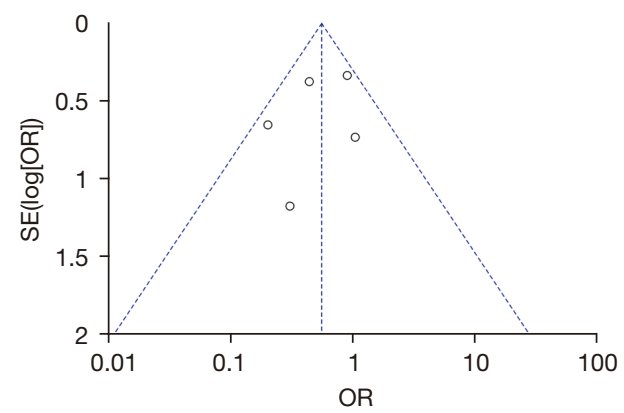

Figure 10 Funnel plot of blood loss. 


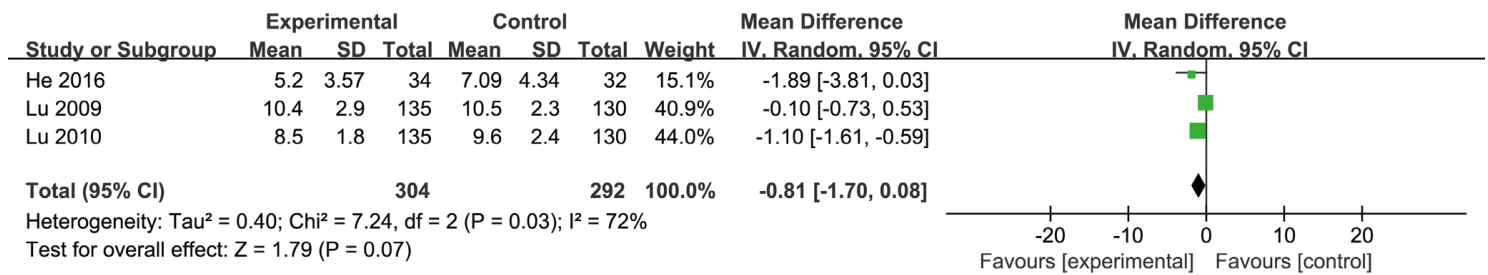

Figure 11 APACHE II scores.

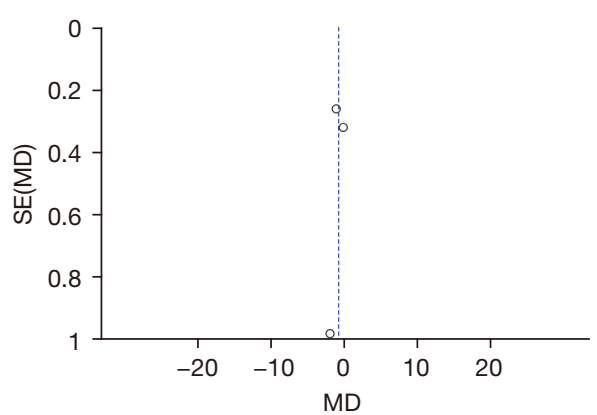

Figure 12 Funnel plot of APACHE II scoring.

of included documents in this study is limited. Subsequent research should increase the number of included documents, so as to increase the sample size and prevent bias in analysis.

In this study, 8 references of comparative study of traditional treatment and intervention of low-molecularweight heparin, which were included in Boolean logic search, were used for meta-analysis, so as to explore the safety and effectiveness of low-molecular-weight heparin on severe acute pancreatitis. The research results show that the MHS between the two groups is statistically heterogeneous $\left(\mathrm{Chi}^{2}=19.59, \mathrm{I}^{2}=95 \%, \mathrm{P}<0.00001\right)$. Fixed-effects model (FEM) analysis showed that the MHS of experimental subjects was obviously shorter than that of controls $(Z=3.24$, $\mathrm{P}=0.001)$; The acute physiology and chronic health score (APACHE II) of the two groups were heterogeneous $\left(\mathrm{Chi}^{2}=7.24, \mathrm{I}^{2}=72 \%, \mathrm{P}=0.03\right)$; No heterogeneity was found in the amount of bleeding $\left(\mathrm{Chi}^{2}=5.83, \mathrm{I}^{2}=31 \%, \mathrm{P}=0.21\right)$, FEM analysis showed the number of complications in the experimental group was significantly less than that in the control group $(\mathrm{Z}=2.70, \mathrm{P}=0.007)$. This may be because the early intervention with low molecular weight heparin in patients of the experimental group effectively prevented and reduced the occurrence of inflammatory reactions in patients and improved the microcirculation state, which had a good treatment effect on patients. Therefore, the average hospitalization time of patients in the experimental group was short. In conclusion, LMH intervention can dramatically reduce the average hospital stay and complications of patients with SAP, improve treatment efficacy, and has high safety.

\section{Conclusions}

Meta-analysis was performed on 8 included studies to investigate the effect of LMH on SAP. LMH intervention can dramatically reduce the average hospital stay and complications of patients with SAP, improve treatment efficacy, with high safety. However, due to confounding factors, the meta-analysis was also limited. The references selected were all case-control studies with survival bias. Moreover, risk factors may not have been included, greatly reducing the size of the pooled effect. There needs to be further exploration of the influence of LMH on the outcomes for patients with SAP, to improve the results. In conclusion, this work provides references for the clinical treatment of pancreatitis.

\section{Acknowledgments}

Funding: None.

\section{Footnote}

Reporting Checklist: The authors have completed the PRISMA reporting checklist. Available at https://dx.doi. org/10.21037/apm-21-3058

Conflicts of Interest: All authors have completed the ICMJE uniform disclosure form (available at https://dx.doi. org/10.21037/apm-21-3058). The authors have no conflicts of interest to declare.

Ethical Statement: The authors are accountable for all aspects of the work in ensuring that questions related 
to the accuracy or integrity of any part of the work are appropriately investigated and resolved.

Open Access Statement: This is an Open Access article distributed in accordance with the Creative Commons Attribution-NonCommercial-NoDerivs 4.0 International License (CC BY-NC-ND 4.0), which permits the noncommercial replication and distribution of the article with the strict proviso that no changes or edits are made and the original work is properly cited (including links to both the formal publication through the relevant DOI and the license). See: https://creativecommons.org/ licenses/by-nc-nd/4.0/.

\section{References}

1. Barkay O, Niv E, Santo E, et al. Low-dose heparin for the prevention of post-ERCP pancreatitis: a randomized placebo-controlled trial. Surg Endosc 2008;22:1971-6.

2. DU JD, Zheng $X$, Huang ZQ, et al. Effects of intensive insulin therapy combined with low molecular weight heparin anticoagulant therapy on severe pancreatitis. Exp Ther Med 2014;8:141-6.

3. Lu XS, Qiu F, Li JQ, et al. Low molecular weight heparin in the treatment of severe acute pancreatitis: a multiple centre prospective clinical study. Asian J Surg 2009;32:89-94.

4. Rabenstein T, Roggenbuck S, Framke B, et al. Complications of endoscopic sphincterotomy: can heparin prevent acute pancreatitis after ERCP? Gastrointest Endosc 2002;55:476-83.

5. Rabenstein T, Fischer B, Wiessner V, et al. Lowmolecular-weight heparin does not prevent acute postERCP pancreatitis. Gastrointest Endosc 2004;59:606-13.

6. Ung KA, Rydberg L, Modin S, et al. A preventive effect of unfractionated heparin on post-ERCP pancreatitis is suggested by positive effects on laboratory markers. Hepatogastroenterology 2011;58:168-73.

7. Lu XS, Qiu F, Li YX, et al. Effect of lower-molecular weight heparin in the prevention of pancreatic encephalopathy in the patient with severe acute pancreatitis. Pancreas 2010;39:516-9.

8. He WH, Yu M, Zhu Y, et al. Emergent Triglyceridelowering Therapy With Early High-volume Hemofiltration Against Low-Molecular-Weight Heparin Combined With Insulin in Hypertriglyceridemic Pancreatitis: A Prospective Randomized Controlled Trial. J Clin Gastroenterol 2016;50:772-8.
9. Qiu Q, Li GJ, Tang L, et al. The efficacy of low molecular weight heparin in severe acute pancreatitis: A systematic review and meta-analysis of randomized controlled trials. J Dig Dis 2019;20:512-522.

10. Oo TH. Comment on "Efficacy of Prophylactic LowMolecular Weight Heparin for Ambulatory Patients With Advanced Pancreatic Cancer: Outcomes From the CONKO-004 Trial”. J Clin Oncol 2016;34:515.

11. Ponert JM, Gockel LM, Henze S, et al. Unfractionated and Low Molecular Weight Heparin Reduce Platelet Induced Epithelial-Mesenchymal Transition in Pancreatic and Prostate Cancer Cells. Molecules 2018;23:2690.

12. Yu Q, Qiu Y, Chen X, et al. Chemotherapy priming of the Pancreatic Tumor Microenvironment Promotes Delivery and Anti-Metastasis Efficacy of Intravenous Low-Molecular-Weight Heparin-Coated Lipid-siRNA Complex. Theranostics 2019;9:355-68.

13. Tozlu M, Kayar Y, İnce AT, et al. Low molecular weight heparin treatment of acute moderate and severe pancreatitis: A randomized, controlled,open-label study. Turk J Gastroenterol 2019;30:81-7.

14. Uyar S, Harmandar F, Kök M, et al. Management of hypertriglyceridemia induced acute pancreatitis and therapeutic plasmapheresis : Report of nine cases and review of literature. Acta Gastroenterol Belg 2017;80:71-4.

15. Kuchay MS, Farooqui KJ, Bano T, et al. Heparin and insulin in the management of hypertriglyceridemiaassociated pancreatitis: case series and literature review. Arch Endocrinol Metab 2017;61:198-201.

16. Song X, Shi D, Cui Q, et al. Intensive insulin therapy versus plasmapheresis in the management of hypertriglyceridemia-induced acute pancreatitis (Bi-TPAI trial): study protocol for a randomized controlled trial. Trials 2019;20:365.

17. Lemke M, Beyfuss K, Hallet J, et al. Patient Adherence and Experience with Extended Use of Prophylactic Low-Molecular-Weight Heparin Following Pancreas and Liver Resection. J Gastrointest Surg 2016;20:1986-96.

18. O'Reilly EM, Barone D, Mahalingam D, et al. Randomised phase II trial of gemcitabine and nabpaclitaxel with necuparanib or placebo in untreated metastatic pancreas ductal adenocarcinoma. Eur J Cancer 2020;132:112-21.

19. Li S, Zhang S, Li R, et al. Prophylactic low-molecularweight heparin administration protected against severe 
acute pancreatitis partially by VEGF/Flt-1 signaling in a rat model. Hum Exp Toxicol 2020;39:1345-54.

20. Degardin J, Pons B, Ardisson F, et al. Multiple organ

Cite this article as: Wang $\mathrm{H}, \mathrm{Xu} \mathrm{Y}$, Yang $\mathrm{M}$, Zheng D, Chen L. Systematic review and meta-analysis of the safety and effectiveness of low molecular heparin for severe acute pancreatitis. Ann Palliat Med 2021;10(11):11695-11704. doi: 10.21037/apm-21-3058 failure complicating a severe acute necrotising pancreatitis secondary of a severe hypertriglyceridemia: a case report. Ann Fr Anesth Reanim 2013;32:611-4. 